\title{
Short pauses while studying considered harmful
}

\section{Leppänen, Leo}

International Academy of Technology, Education and Development 2016

Leppänen , L , Leinonen , J \& Vihavainen , A 2016 , Short pauses while studying considered harmful . in EDULEARN16 Proceedings . EDULEARN proceedings, International Academy of Technology, Education and Development, pp. 1900-1904, International Conference on Education and New Learning Technologies, Barcelona, Spain , 04/07/2016 . https://doi.org/10.21125/edulearn.201

http://hdl.handle.net/10138/316021

https://doi.org/10.21125/edulearn.2016.1376

acceptedVersion

Downloaded from Helda, University of Helsinki institutional repository.

This is an electronic reprint of the original article.

This reprint may differ from the original in pagination and typographic detail.

Please cite the original version. 


\title{
SHORT PAUSES WHILE STUDYING CONSIDERED HARMFUL
}

\author{
L. Leppänen, J. Leinonen, A. Vihavainen \\ University of Helsinki (FINLAND) \\ leo.leppanen@helsinki.fi, juho.leinonen@helsinki.fi, avihavai@cs.helsinki.fi
}

\begin{abstract}
Study and time management skills are an integral part of successful learning. Taking pauses and spacing out learned content over time helps assimilate knowledge and improves long term retention of information. In this study, we explore pauses in learning and their effect on course outcomes. Our research indicates that pauses between 5 seconds and 5 minutes in length are harmful for course outcomes. Analysis of student backgrounds indicates that previous experience with the subject is negatively correlated with the amount of harmful pauses.
\end{abstract}

Keywords: pauses, learning, programming

\section{INTRODUCTION}

Students work in environments riddled with distractions. Phones in their pockets keep them always connected to their peers and friends. Even some of their learning tools provide a constant source of distractions: it's easy to get off-task when working or taking notes on a computer. The students check their phones or social media accounts while making notes, play a quick round of a popular online game during "the boring part" of the lecture or just end up going "off-topic" while researching a paper. All these distractions essentially constitute multi-tasking and and task switching, both of which have been previously linked to increased cognitive loads and worse learning outcomes $[4,7,8]$.

Yet not all pauses are bad for the student. Research has also shown the value of spacing out learning: it is better for retention to study something in multiple segments than it is to "crunch" for the same total duration of time [3]. Spacing out work also tends to lead to students beginning their work earlier, which in turn, potentially reduces procrastination and helps with the classic problem starting coursework too close to a deadline [9].

In this paper, we look at how the pauses students take from programming tasks affect their exam scores. We view programming as essentially the pathological case for distractions: students have to work on a computer and constantly check online material for help. They often work from home, surrounded by activities they most likely would prefer over studying. Even school computer facilities are filled with distractions both in form of other students and the internet.

While our research is restricted to this specific context, we see this as something that generalizes to other situations: laptops are no longer uncommon in lecture halls and course work is often completed or at least turned in into an electronic learning environment such as Moodle.

\section{PREVIOUS WORK}

The previous work on the effect of pauses shows mixed results. Some research indicates that taking pauses can be beneficial for the task that is currently ongoing [2,3]. However, especially multitasking [TODO: viittaa], which can be seen as taking repeated pauses from a primary task in order to engage other concurrent tasks, has been shown to have a negative effect on learning $[7,8]$.

Smith and Blankenship have studied pausing behaviour in learning and found effects they labeled fixation and incubation [2]. Fixation is a state which a student gets "railroaded" into an initial problem solving strategy that is not beneficial for solving the task. Spending time off-task can have the effect of undoing such fixation. Incubation on the other hand is the phenomena of being able to more easily solve a problem after letting it "sit" for a while while engaging in off-task behaviour. Incubation is also known in the popular parlance as "sleeping on a problem", but does not necessarily require actual sleep or long periods of time. 
Dempster has studied the spacing effect in learning [3]. He discovered that students who study in multiple sessions performed better in a test compared to a group of students who studied in a single session. This indicates that it is better to space out learning into multiple study sessions instead of "crunching" in a single session.

On the other hand, multitasking has been shown to affect performance negatively in multiple contexts. Junco and Cotten showed that using Facebook and sending text messages while studying had a negative associated with college students' GPAs [7]. Wood et al. compared the use of social media with note-taking and showed that students who used social media performed worse than students who didn't use any technology during lectures [6]. Rosen et al. showed that students who texted excessively during lectures (more than 15 text messages sent or received) performed around $10 \%$ worse than students who texted less [4]. Hembrooke and Gay examined laptop use on lectures and found that students who were able to use a laptop performed worse than a control group who were told not to use a laptop [5].

\section{RESEARCH QUESTIONS}

In this paper we seek to answer two research questions:

1. Is it possible to determine certain pause lengths as either beneficial or harmful for learning?

2. Are students' pausing behaviours correlated with student demographics?

As previous work is conflicted on whether pauses are beneficial or not, we explore the effect of different kinds of pauses on learning with the first research question. With the second research question, we are interested in looking for correlations between pausing behaviour and student demographics. Knowing the pausing behaviour for different demographic groups and the effect of pausing behaviour on learning would help in researching the causes of the possible beneficial or detrimental effect of pauses as well as yield the possibility of guiding certain demographic groups to a better way of studying.

\section{METHODOLOGY}

The data for the experiment comes from a introductory programming course organized during fall 2014 by the University of Helsinki Department of Computer Science. The course is a compulsory bachelor's level course for students of the department, but many participants are majoring in other subjects.

The participants used an instrumented programming environment [1] that stored fine-grained data from the learning process, including timestamps of each key press as well as details on the assignments that the participants were working on. In addition, background information on the participants was acquired from questionnaires embedded in the online material. Participants were rewarded with course points for answering the questionnaires. Our data also included details on how they fared on a course final pen-and-paper exam.

Participants that declined to answer the background survey, did not provide us with the fine-grained data or did not attend the course exam were excluded from the analysis. After these exclusions, our data set consisted of $n=156$ participants. Key demographics of the included participants are detailed in Tables 1,2 and 3.

\begin{tabular}{c|c|c|c|c} 
Total & Male & Female & $\begin{array}{c}\text { Year of birth } \\
\text { (min - median - max })\end{array}$ & $\begin{array}{c}\text { Handedness } \\
\text { (left - right - other) }\end{array}$ \\
\hline 156 & 106 & 50 & $1970-1993-1996$ & $15-150-1$
\end{tabular}

Table 1: Key participant demographics 


\begin{tabular}{c|ccc|cc} 
Total & \multicolumn{4}{|c|}{ Programming background } & \multicolumn{2}{c}{ Background in the course language } \\
& None & Some & Plenty & No & Yes \\
\hline 156 & 91 & 33 & 32 & 119 & 37
\end{tabular}

Table 2: Participant backgrounds in programming

\begin{tabular}{c|c|c|c|c|c|c} 
Total & $\begin{array}{c}\text { Compre- } \\
\text { hensive }\end{array}$ & $\begin{array}{c}\text { Upper } \\
\text { Secondary }\end{array}$ & $\begin{array}{c}\text { University of } \\
\text { applied sciences }\end{array}$ & Bachelor's & Master's & $\begin{array}{l}\text { Doctorate or } \\
\text { licentiate }\end{array}$ \\
\hline 156 & 0 & 123 & 4 & 21 & 8 & 0
\end{tabular}

Table 3: Highest level of finished education

Within the framework of the seven week courses, the participants would complete up to 176 programming assignments. In our analysis, we considered the programming assignments the "primary task". All other tasks were considered "secondary tasks". Secondary tasks would include other learning related activities such as reading the online course material, attending lectures, etc.; as well as non-learning related activities such as sleeping, eating, and socialising.

Based on this clear distinction between the primary task and secondary tasks, we determined each key press in our data as a unit of work. This naturally leads to a definition of pause as a period of time between two units of work. This definition of a "pause" is quite strict, and thus, we combined units of work where the pause between the two adjacent units of work was less than a second. This leaves us with the following definition for a pause: a pause is a time period longer than or equal to 1 second between two subsequent keypress events.

Pauses were then divided into overlapping buckets based on the pause lengths. The buckets used were as follows: single second buckets from one second to 20 seconds; 5 -second buckets from 1 seconds to 60 seconds; 10 second buckets from 1 second to 2 minutes; 1 minute buckets from 1 minute to 20 minutes; 5 minute buckets from 5 minutes to 45 minutes; as well as buckets for 1-2 hour, 2-6 hour, 12-24 hour and 24+ hour pauses.

For each of the buckets, the relative amount of each participant's total pauses that belonged into said bucket were then subjected to a correlation analysis with the participant's exam score and background variables.

\section{RESULTS}

\subsection{Research question 1}

Statistically significant $(p<0.05)$ Pearson correlations were found between certain pause lengths and exam scores: Pauses between 5 seconds and 5 minutes were found to be statistically significantly negatively correlated with the exam scores, even after a Bonferroni correction was applied to account for the high number of correlation factors calculated.

Each of the buckets with a statistically significant negative correlations had a slightly different correlation coefficient, but the harmful pauses explain on average $11.5 \%$ of the variance of the participants' exam scores.

Furthermore, a positive correlation ( $r=0.44, p<0.01$, bonferroni corrected) between extremely short pauses of one to two seconds and exam scores was found.

\subsection{Research question 2}


Having identified certain types of pauses as harmful, we searched for correlations between relative counts of harmful pauses and student demographics.

No statistically significant Bonferroni corrected correlations were found between relative count of harmful pauses and student handedness, educational background, year of birth or the first year of studies in the university.

At the same time, statistically significant correlations were found between relative amount of harmful pauses and student programming background in general $(r=-0.22, p=0.02)$, student programming background in the course's programming language of choice $(r=-0.38, p<0.01)$.

\section{DISCUSSION}

Our results indicate that those who continuously work on the task at hand without significant pauses perform well, even when the work may not immediately lead to expected outcomes. Participants who take either a relatively large amount of 5 second to 5 minute pauses perform poorer than others. This effect is apparent even without knowing what the students are doing at the time, which suggests that what is done during the pause does not matter -- participants may e.g. be looking at course materials or non-relevant content on the internet.

Interpretation of the positive correlation with extremely short 1-2 second pauses and exam scores is curious and has two possible interpretations: either these pause lengths are beneficial by themselves, or alternatively they are rather indicative of a lack of otherwise harmful pauses. Without further research, we are inclined to take the more safe view of this effect stemming from the lack of a harmful effect rather than from some independent positive effect.

The results provide evidence that the worst approach to studying is to continuously switch tasks, and supports the wider literature on cognitive load theory and task switching as well as the spacing effect. Based on our observations, the most beneficial strategy regarding pauses is to focus on one single thing at a time, and if facing a challenge, fighting it out. It would seem that switching between the primary task at hand (such as working on a programming assignment) and a supporting task (such as reading course material) can be harmful if the participant constantly changes between these tasks, spending only very short amounts of time on one before switching again.

Our study has multiple limitations, the primary being that our data is from a single context with a highly specific pedagogical methodology. Previous research has shown that even among the courses of this specific subject, large differences are present [10]. Furthermore, the participants are from a largely homogenous background. These facts might limit how well these result generalize to other subjects and contexts.

Another limitation is that we are unable to distinguish between "beneficial" off-task behaviour such as reading the course material and "harmful" off-task behaviour such as texting. Controlling for this would require

More broadly, this work suggests that the design of learning activities and environments should attempt to minimize short distractions. In an electronic environment, this would likely entail automatically detecting and correcting this kind of harmful behaviour detailed above and structuring the learning activity so that the participants focus on only one cognitively demanding activity at a time. Activities should be designed to avoid situations where a participant is (near)simultaneously completing assignments and browsing course material. Instead of just quickly checking for a fact from the material, it is more beneficial to refresh a longer section of the material.

The correlations between harmful pauses and participant background variables are mostly as expected. It is logical to expect that a student with extensive background in a task would be able to complete said task with less pauses taken to, for example, check an accompanying study material. It 
is similarly logical that the same effect is visible in students that have background in similar tasks, even if they are not familiar with the exact subject.

An interesting avenue of future research is whether the students' cognitive flexibility affect the effects of harmful pauses. Similarly, future work should investigate whether more complex background variables such as student motivation are correlated with the habit of taking harmful pauses.

\section{REFERENCES}

[1] Vihavainen, A.; Vikberg,T.; Luukkainen, M. and Pärtel, M. (2013). Scaffolding students' learning using test my code. In Proceedings of the 18th ACM conference on Innovation and technology in computer science education (ITiCSE '13). ACM, New York, NY, USA, pp. 117-122.

[2] Smith, S. M., \& Blankenship, S. E.. (1991). Incubation and the Persistence of Fixation in Problem Solving. The American Journal of Psychology, 104(1), 61-87. http://doi.org/10.2307/1422851

[3] Dempster, F. N. (1988). The spacing effect: A case study in the failure to apply the results of psychological research. American Psychologist, 43(8), 627.

[4] Rosen, L. D., Lim, A. F., Carrier, L. M., \& Cheever, N. A. (2011). An empirical examination of the educational impact of text message-induced task switching in the classroom: Educational implications and strategies to enhance learning. Psicología educativa, 17(2), 163-177.

[5] Hembrooke, H., \& Gay, G. (2003). The laptop and the lecture: The effects of multitasking in learning environments. Journal of computing in higher education, 15(1), 46-64.

[6] Wood, E., Zivcakova, L., Gentile, P., Archer, K., De Pasquale, D., \& Nosko, A. (2012). Examining the impact of off-task multi-tasking with technology on real-time classroom learning. Computers \& Education, 58(1), 365-374.

[7] Junco, R., \& Cotten, S. R. (2012). No A 4 U: The relationship between multitasking and academic performance. Computers \& Education, 59(2), 505-514.

[8] Monsell, S. (2003). Task switching. Trends in cognitive sciences, 7(3), 134-140.

[9] Martin, J., Edwards, S. H., \& Shaffer, C. A. (2015, July). The Effects of Procrastination Interventions on Programming Project Success. In Proceedings of the eleventh annual International Conference on International Computing Education Research (pp. 3-11). ACM.

[10] Vihavainen, A., Airaksinen, J., \& Watson, C. (2014, July). A systematic review of approaches for teaching introductory programming and their influence on success. In Proceedings of the tenth annual conference on International computing education research (pp. 19-26). ACM. 